than is usually understood. The stability of such organizations as the Army, church, and even trades unions, is only a reflexion of the family constellation, and this is exemplified by such terms as 'brother officers' and the use of the appellations 'father' and 'brother' in church and trades unions. The respect for authority and the manner in which we regard the State are only projections of our feelings derived from the family.

We spend a tremendous amount of money, both directly and indirectly, on broken marriages, divorce and their consequences : delinquency, neurosis, and, in some cases, psychosis. We cannot know too much about the nature of marriage and why it breaks down. The book is well written, with a good index, and none of the authors breaks out in the too blatant Americanisms which distract and annoy the British reader.

Clifford Allen

\section{CHEMICAL BASIS OF BLOOD GROUP SPECIFICITY}

\section{Blood Group Substances}

Their Chemistry and Immunochemistry. By Elvin A. Kabat. Pp. ix +330 . (New York: Academic Press, Inc.; London: Academie Books, Ltd., 1956.) 8 dollars.

WE greatly increased use of blood transfusion in medical practice during the past two decades has led to a rapid expansion in our knowledge of human blood-groups, and nine independent systems have been identified by serological methods. An understanding of the chemical basis of blood-group specificity has, however, lagged far behind the serological discoveries and even now it is not possible to assign a detailed chemical structure to any one blood group substance, or to define completely the smaller structural units within the complex macromolecules which confer on them their characteristic serological properties. Much progress towards this goal has nevertheless been achieved, and the present book by Elvin Kabat represents the first attempt to bring together all the existing information on the chemistry and immunochemistry of the blood group substances.

Isolation of the materials responsible for bloodgroup specificity from the red cell surface presents considerable technical difficulties and for this reason investigations on the chemical nature of the blood group substances have been almost entirely restricted to those group-specific materials which also occur in tissue fluids and secretions in a water-soluble form, that is, to the $\mathrm{A}, \mathrm{B}, \mathrm{O}(\mathrm{H})$ and Lewis $\left(\mathrm{Le}^{\mathrm{a}}\right)$ substances. The major part of the book is devoted to a comprehensive survey of the methods used for the isolation, purification and chemical and immunochemical analyses of these water-soluble mucopolysaccharides. The author's own outstanding contribution to the immunochemistry of blood group substances has been the application to them of the quantitative precipitin technique developed by Heidelberger and his co-workers for the quantitative measurement of antigens, antibodies and complement, and the emphasis throughout the book is on this approach to blood group studies. Not all readers will agree with the author's low estimate of the value of results obtained by the hæmagglutination technique, which, although admittedly less precise, has a greater sensitivity than the precipitin method. Indeed the first indications of the simple structures responsible for specificity in both $\mathrm{A}$ and $\mathrm{H}$ substances were obtained by means of hæmagglutination inhibition experiments. The amount of experimental detail included seems rather excessive in some parts of the text and many of the long tables, which have been taken unchanged from the original papers, could with advantage have been given in a summarized form. The author has, however, reviewed the field with admirable thoroughness, the presentation is accurate and systematic and each chapter contains an extensive list of references.

This is essentially a book for the specialist and little concession is made to the general reader. It should nevertheless prove invaluable as an introduction for those entering the field of chemical studies on bloodgroup substances and as a reference book for immunochemists and biochemical geneticists interested in these serologically active mucopolysaccharide materials which are such excellent 'markers' in the field of human genetics. WINIFRED M. WATKINS

\section{LIGHT-SCATTERING AND MACROMOLECULAR CHEMISTRY}

Light-Scattering in Physical Chemistry

By Dr. K. A. Stacey. Pp. viii +226 . (London: Butterworths Scientific Publications; New York: Academic Press, Inc., 1956.) 40s.

TN 1944 Debye showed that the molecular weight and dimensions of a polymer in solution can be determined by the light-scattering technique. Since then a large number of papers on the application of this method to the study of macromolecules have appeared, and it is becoming increasingly popular as a routine technique for determining the weight. average molecular weights of polymers. Dr. Stacey is the first worker to devote an entire book to a survey of this new and important field, and in doing so he makes a timely contribution to the existing literature.

The book falls roughly into two equal parts, one dealing with the theory and practice of light-scattering relevant to this particular application, and the other with its use in the study of macromolecules. The first part is prefaced by a summary of the various methods for determining molecular weights of large molecules, and then the theory of light-scattering is developed in a way which emphasizes clearly the underlying principles. There is, however, a tendency to include short summaries of a large number of specific extensions of the theory when a more careful selection of the material would have allowed an improved presentation of the most important contributions. This half of the book is concluded by a very useful account of modern experimental methods. The remaining chapters are reserved for a review of the applications of this method in high polymer, protein and polyelectrolyte chemistry. Here again a large number of papers have been summarized, but little attempt is made to correlate or appraise the various contributions.

The author maintains that the time is not ripe for an authoritative monograph, but one feels that a more critical assessment of the present status of the light-scattering technique is required. The book does, however, contain some five hundred references to original papers published prior to 1956 and it will be of considerable value to anyone connected with the
field. 